\title{
Molecular detection of porcine Enterocytozoon bieneusi infection in Peninsular Malaysia and epidemiological risk factors associated with potentially zoonotic genotypes
}

\begin{abstract}
Enterocytozoon bieneusi is an emerging opportunistic pathogen infecting humans, and both domestic and wild pigs are known to harbour zoonotic genotypes. There remains a paucity of information on the prevalence and epidemiology of this enteropathogen in Southeast Asia. The present study was undertaken to determine the molecular prevalence and risk factors associated with E. bieneusi infection among commercially farmed pigs in Malaysia. Faecal samples were collected from 450 pigs from 15 different farms and subjected to nested PCR amplification of the ribosomal internal transcribed spacer (ITS) gene of E. bieneusi. Phylogenetic analysis involved 28 nucleotide sequences of the ITS region of E. bieneusi. An intervieweradministered questionnaire provided information on the animal hosts, farm management systems and environmental factors and was statistically analysed to determine the risk factors for infection. The prevalence of E. bieneusi infection was relatively high $(40.7 \%)$. The highest prevalence $(51.3 \%)$ was recorded among the piglets, while the adults showed the lowest level of infection (31.3\%). Multivariate analysis indicated that age of the pigs, distance of the farm from human settlement and farm management system were significant risk factors of infection. Three genotypes (EbpA, EbpC and Henan-III) detected among the pigs are potentially zoonotic. The high prevalence of E. bieneusi among locally reared pigs, the presence of zoonotic genotypes and the spatial distribution of pig farms and human settlements warrant further investigation on the possibility of zoonotic transmission.
\end{abstract}

Keyword: Enterocytozoon bieneusi; ITS; Malaysia; Pigs; Zoonosis 